\title{
Erratum to: Snowstorm testes
}

\author{
Eduardo Kaiser Ururahy Nunes Fonseca $\odot,{ }^{1}$ Fernando Ide Yamauchi, ${ }^{1,2}$ \\ Leonardo Lima Borges, ${ }^{3,4}$ Miguel José Francisco Neto, ${ }^{1}$ Ronaldo Hueb Baroni ${ }^{1}$ \\ ${ }^{1}$ Imaging Department, Hospital Israelita Albert Einstein, Av. Albert Einstein, 627, São Paulo 05652-901, Brazil \\ ${ }^{2}$ INRAD, HC-FMUSP, R. Dr. Ovídio Pires de Campos, 75, São Paulo 05403-010, Brazil \\ ${ }^{3}$ Urology Department, Hospital Israelita Albert Einstein, Av. Albert Einstein, 627, São Paulo 05652-901, Brazil \\ ${ }^{4}$ Instituto do Câncer do Estado de São Paulo (ICESP) - FMUSP, Av. Dr. Arnaldo, 251, São Paulo 01246-000, Brazil
}

\section{Erratum to:Abdom Radiol (2017) DOI: $10.1007 / \mathrm{s} 00261-017-1110-y$}

In the published version of this article Figs. 1 and 2 were interchanged. The correct images are as follows:
The online version of the original article can be found under doi: 10.1007/s00261-017-1110-y.

Correspondence to: Eduardo Kaiser Ururahy Nunes Fonseca; email: edukaiser_unf@hotmail.com

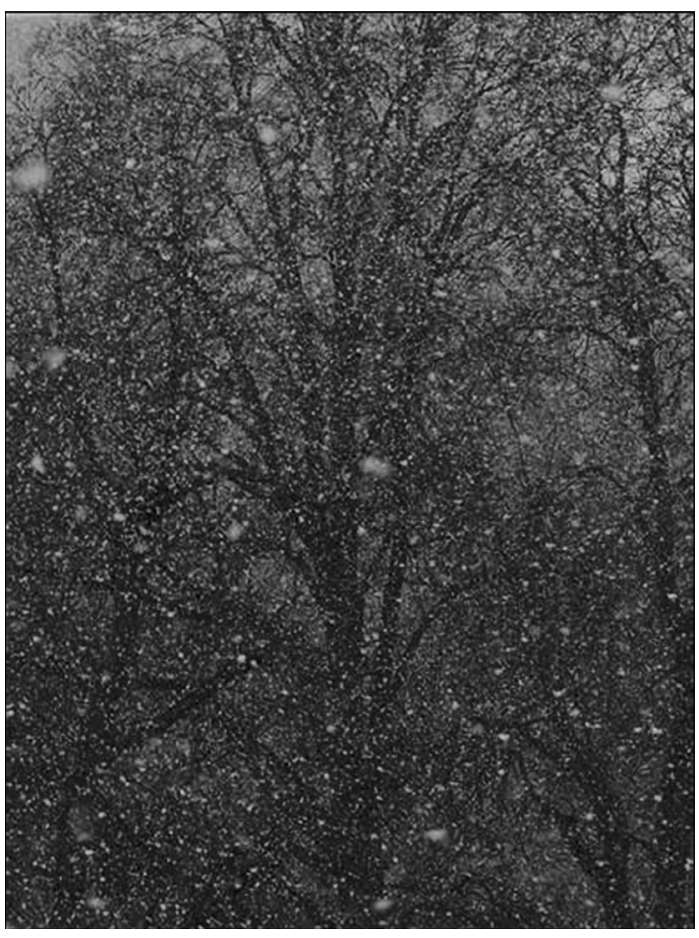

Fig. 1. Falling snow. Downloaded from https://pixabay. com/en/blizzard-snow-flurry-snowflakes-91900/. 


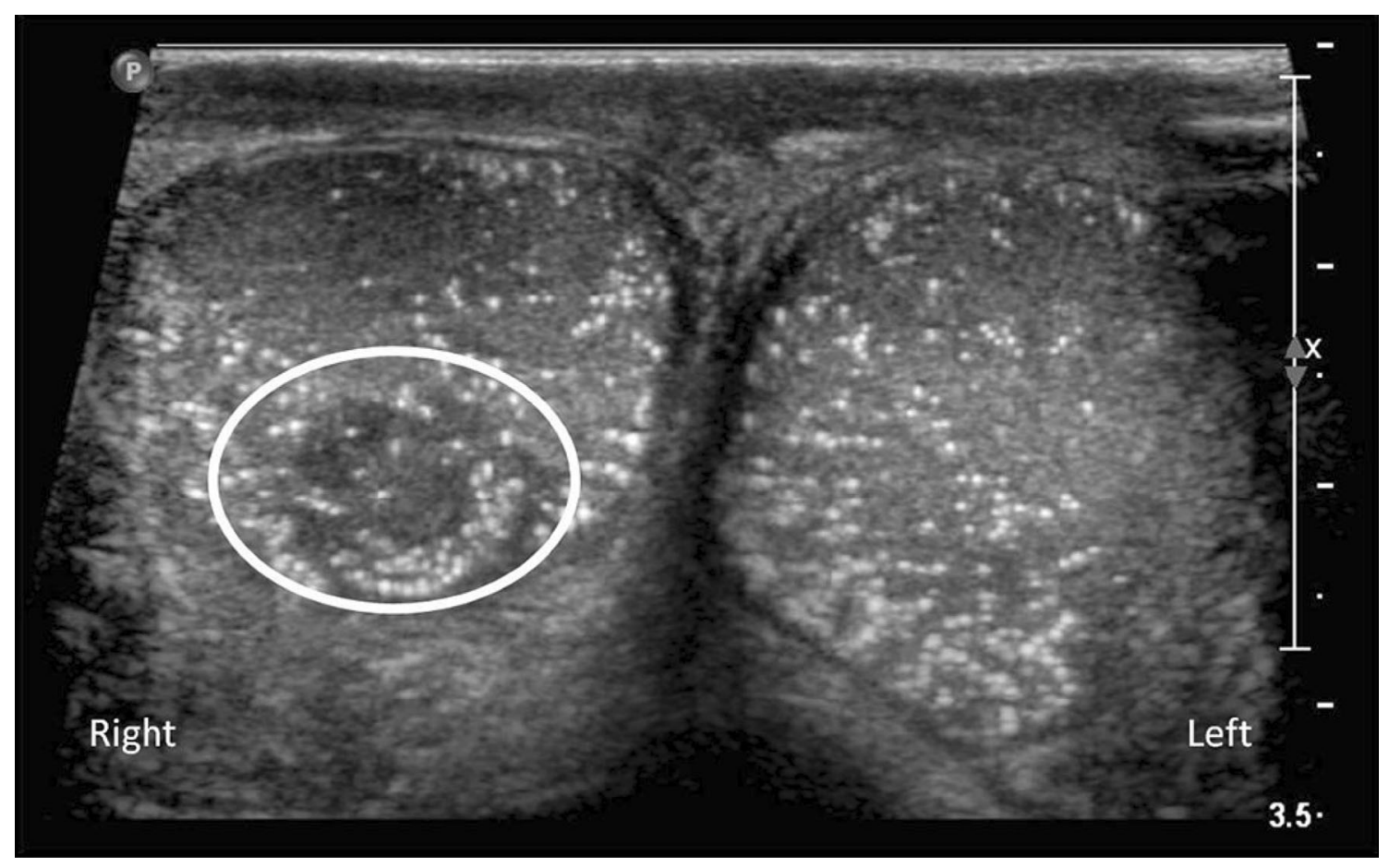

Fig. 2. Ultrasonography in transverse view showing innumerable tiny echogenic foci scattered within the parenchyma of both testes, producing "snowstorm testes." A hypoechoic nodule (circle) is seen in the right testicle, proven at pathology to be a seminoma. 